\title{
TEMPERATURE DEPENDENCE OF MAGNETO-OPTICAL EFFECT IN MAGNETIC FLUIDS
}

\author{
S. TAKETOMI*, M. UKITA, H. MIYAJIMA, and S. CHIKAZUMI \\ Dept. of Phys., Keio University, Hiyoshi, Yokohama 223, Japan \\ * Matsumoto Yushi-Seiyaku Co., Ltd., Yao, Osaka 581, Japan
}

\begin{abstract}
Temperature dependence of magnetic birefringence of magnetic fluids under a magnetic field $\mathrm{H}$ was studied. Temperature dependence of the phase difference $\theta$ between the two modes of the rays with polarization parallel and perpendicular to the field $\mathrm{H}$ was found to obey the generalized CurieWeiss law, or $\theta \propto\left(T-T_{0}(H)\right)$ where $T$ is the temperature of the magnetic fluid. The function $T_{0}$ of $\mathrm{H}$ satisfies the empirical formula, $\mathrm{T}_{0}(\mathrm{H}) \boldsymbol{\alpha} \exp (-\alpha \mathrm{H})$, where $\alpha$ is a constant.
\end{abstract}

\section{INTRODUCTION}

Magnetic fluid is a colloidal solution containing magnetic colloidal particles[1]. It has many interesting properties such as strong magnetization in spite of the liquid, a large magneto-optical effect etc.. In this paper, the magnetic birefringence, especially, its temperature dependence is discussed.

The magnetic fluid is an opaque liquid. It becomes, however, transparent when it is diluted or it is formed in a thin film form of about tens micron thickness. Several authors have been studied the magneto-optical effect of the diluted magnetic fluids[2-10]. On the other hand, one of the present authors reported that the thin film of conc magnetic fluid shows a large magnetic birefringence, which is about $10^{7}$ times larger than that of nitrobenzene[11]. We have already found some interesting characteristics of the effects[12],[13].

one characteristic is that there are two types of field dependences of dichroism in the magnetic fluid.

The second characteristic is that a scaling law exists between the field $H$ and the phase difference $\theta$ between ordinary and extraordinary rays. Here, the ordinary and the extraordinary rays are those having electric field vector perpendicular and parallel to the applied field, respectively. When $\theta$ and $H$ are normalized by proper constants $\theta_{0}$ and $H_{0}$, respectively, the reduced phase difference

$$
\zeta=\theta / \theta_{0}
$$

and the reduced field

$$
h=\mathrm{H} / \mathrm{H}_{0} \text {, }
$$

satisfy a universal function $f_{u}(x)$ by

$$
\zeta=f_{u}(h),
$$

irrespective of the kinds of the magnetic fluids.

The third characteristic is that a scaling law similar to the $\theta-H$ curve holds for the magnetization curve $\mathrm{M}-\mathrm{H}$. Namely when the magnetization $\mathrm{M}$ of the magnetic fluid is normalized by a proper constant $\mathrm{M}_{0}$, the reduced magnetization

$$
m=M / M_{0} \text {, }
$$

and the reduced field $h$ also satisfy the universal function $f_{u}(x)$ given by

$$
m=f_{u}(h) \quad \text {. }
$$

All these experimental results have been obtained at room temperature. In this paper, the experiment was extended to various temperatures. 


\section{EXPERIMENT}

Figure 1 shows the experimental arrangement. The linearly polarized ray enitted from the $5 \mathrm{~mW} \mathrm{He}-\mathrm{Ne}$ laser is introduced to the magnetic fluid thin film through a polarizer. A magnetic field is applied to the sample perpendicular to the light propagation by a Weiss-type magnet. The transmitted light from the sample passes through the analyzer and the intensity

is measured by a photo-detector. Varying the polarization direction of the incident light, the transmissivities of the ordinary and the extraordinary rays are measured. The phase difference $\theta$ is also measured. The details of the measuring techniques are referred to Ref.13.

The construction of the magnetic fluid sample is shown in Fig.2. A 5 mm square window is bored in the polyester thin film of $50 \mu \mathrm{m}$ thickness which is sandwiched with two plane glass plates and this window is filled with the magnetic fluid. The magnetic fluid thin film is thus formed in the window. Its thickness is assumed to be the same as that of the polyester film, $50 \mu \mathrm{m}$ thick.

The colloidal particles and the solvent of the magnetic fluid are ferrite and alkylnaphthalene, respectively. The volume fraction of the colloidal particles is 0.052 . The diameter of the particles distributes widely in log-normal distribution at about $100 \AA$ as a center. Here the lognormal distribution means that the number density of the colloidal particles distributes in Gaussian form with respect to the logarithm of the radius of the particle.

To perform the experiment as a function of temperatures, the sample is clad with a thermojacket, the structure of which is shown in Fig.3. It is composed of two copper plates. A thermistor and electric heaters are installed to controll the temperature of the sample.

The experiment was carried at the temperatures of $289,308,332$ and $363 \mathrm{~K}$. The phase difference $\theta$ is measured as a function of the applied field at each temperature.

\section{RESULTS AND DISCUSSIONS}

From the experiments, it is revealed that the phase difference $\theta$ obeys the generalized CurieWeiss law at a fiexed applied field, i.e.,

$$
\theta \propto\left\{\mathrm{T}-\mathrm{T}_{0}(\mathrm{H})\right\}^{-1}
$$

where $T_{0}(H)$ is a function of $H$ but independent of absolute temperature $T$.

Figuxe 4 shows several examples of the experimental data. The inverse of $\theta$ is plotted as a function of temperature. At fixed fields $H=0.5,0.6,0.7,0.8$ and 0.9 kOe, the inverse of $\theta$ lines up on straight line. The $T_{0}(H)$ is the T value where the extrapolated $\theta^{-1}-T$ curve crosses the abscissa. Figure 5 shows the $H$ dependence of $T_{0}(H)$ in a logarithmic scale.
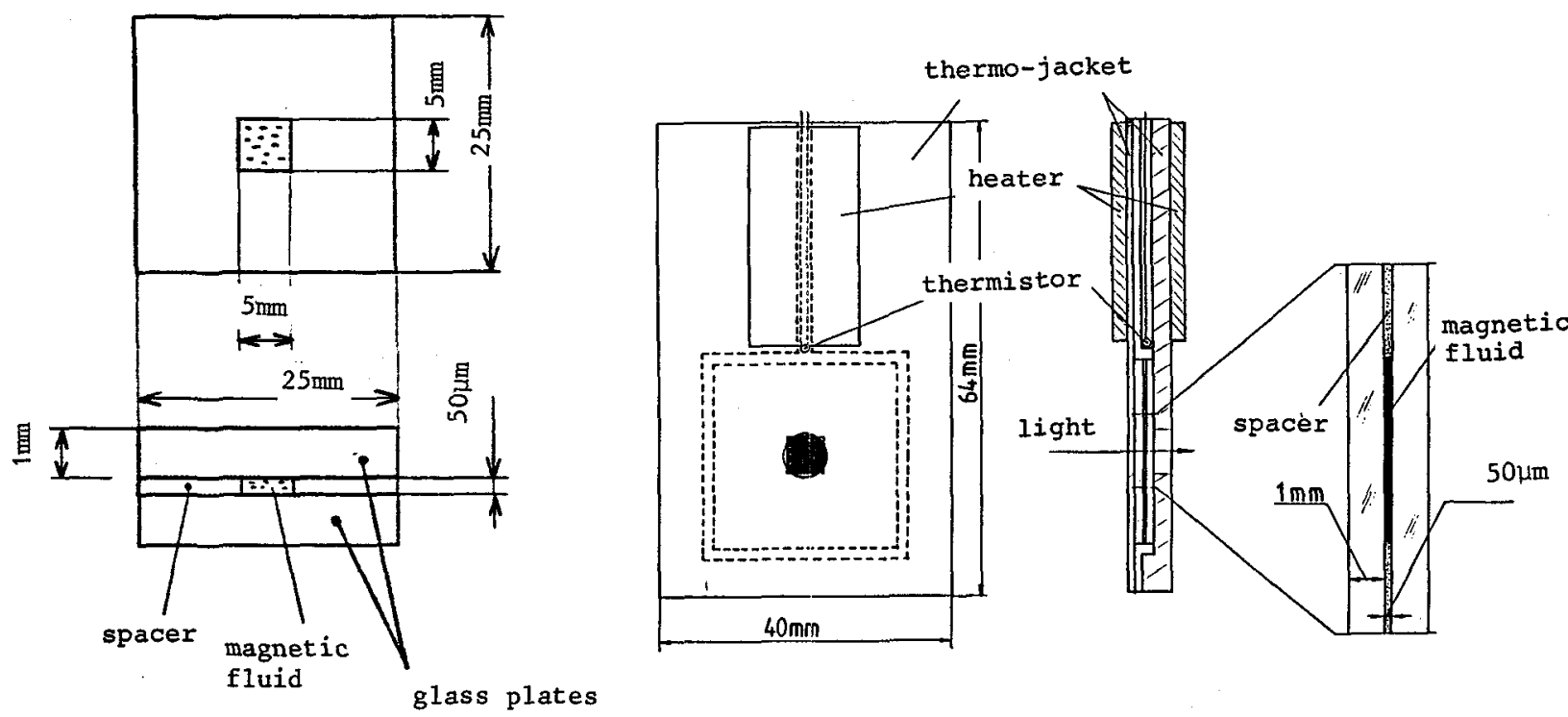

Fig.3 Structure of the thermo-jacket.

Fig.2 Construction of the magnetic fluid sample. 
From these data, the empirical formula

$$
\mathrm{T}_{0}(\mathrm{H}) \propto \exp (-\alpha \mathrm{H}) \text {, }
$$

is obtained, where $\alpha$ is a constant.

We succeeded in interpreting dichroism, birefringence and their mutual relationship by a following physical model[13]. We also interpret the temperature dependence

of $\theta$ by the same model. When the field is applied, a part of the colloidal particles coagulates to form linear chains directed parallel to the field direction. (See Fig.6.) Though the size of the colloidal particles distributes widely in log-normal distribution, let us assume the colloidal particles be the spheres of the same radius D. The numbers of clusters consisting of $k$ colloidal particles is denoted by $\mathcal{L}$. In the following, the cluster of the colloidal particles is assumed to be an elongated ellipsoid for the convenience of the analysis[14].(See Fig.6) The depolarization factor in the field direction of the cluster of $\mathrm{k}$ particles is denoted as $\mathrm{N}_{\mathrm{k}}$. Then the average of $\mathrm{N}_{\mathrm{k}}$ is defined

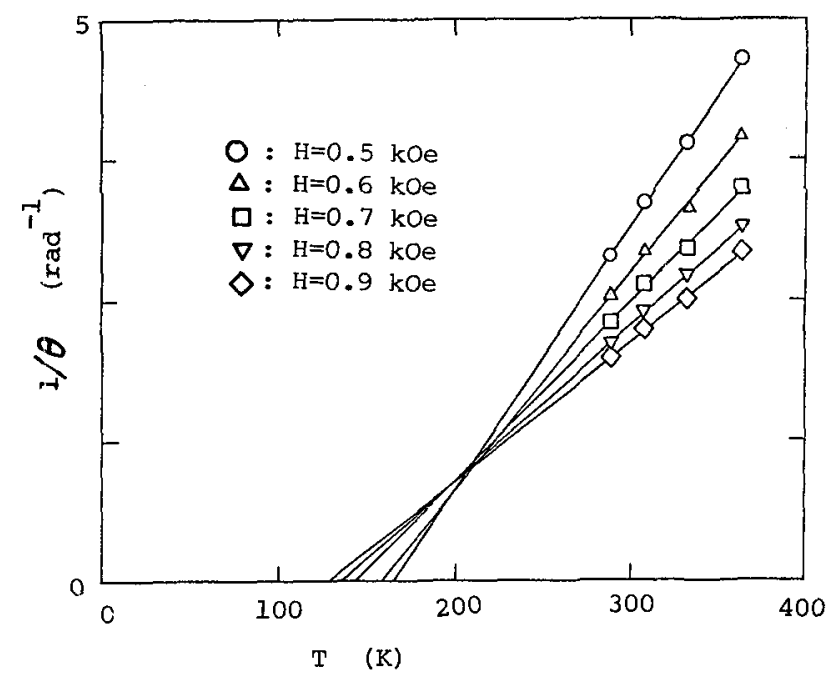

$$
\langle\mathrm{N}\rangle=\phi_{\mathrm{M}}^{-1} \sum_{\mathrm{k}=1}^{\infty} \mathrm{kv}_{0} \mathrm{v}_{\mathrm{k}} \mathrm{N}_{\mathrm{k}},
$$

where $v$ is the volume of the colloidal particle and $\phi_{\text {is }}$ is the volume fraction of the colloidal particles. The number of colloidal particles $n_{0}$ in unit volume of the magnetic fluid satisfies

$$
\phi_{\mathrm{M}}=\mathrm{n}_{\mathrm{O}} \mathrm{V}
$$

The wavelength of the light used in this experiment is $633 \mathrm{~nm}$ while the sizes of the colloidal particles are about $10 \mathrm{~nm}$. The averaged size of the clusters is also a few hundred angstrom. Therefore, the averaged dielectric constant is applicable for the calculation of the refractive index of magnetic fluids for the visible light. Then the relative dielectric constants parallel and perpendicular to the field, $\varepsilon_{/ /}, \varepsilon_{\perp}$, are given by[13]

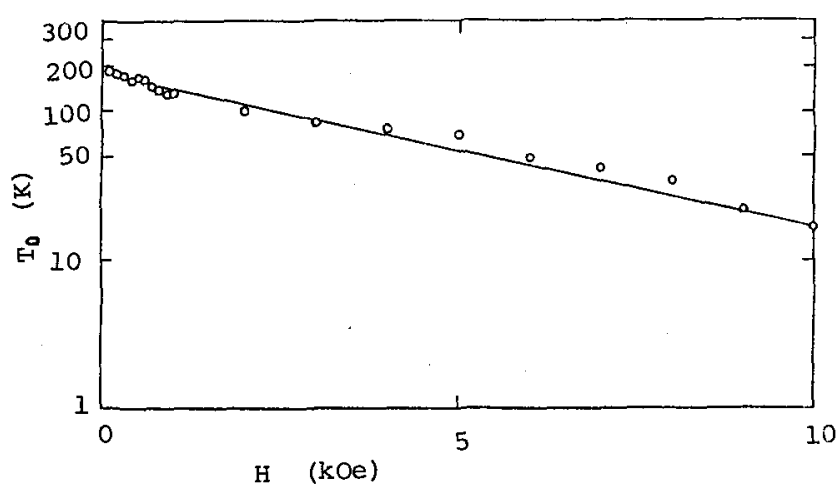

Fig.5 $\mathrm{T}_{\mathrm{g}}(\mathrm{H})$ v.s. H. Logarithms of $\mathrm{T}_{\mathrm{O}}(\mathrm{H})$ is scaled in the ordinate. $\uparrow_{\text {Field } \overrightarrow{\mathrm{H}}}$

$k=1$

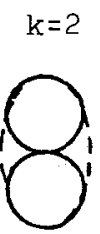

$k=3$

$k=k$

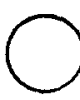

$N_{1}$

$\mathrm{N}_{2}$

$\mathrm{N}_{3}$

$\mathrm{N}_{\mathrm{k}}$

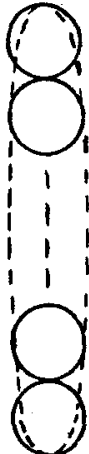

$v_{1}$

$v_{2} \quad v_{3}$

$v_{\mathrm{k}}$

Fig.6 Formation of the chain-like clusters under the field.

The values $\mathcal{H}_{\mathrm{k}}$ and $\mathrm{N}_{\mathrm{k}}$ represent the number of the clusters containing $k$ colloid particles and the depolarization factor of them in the field direction, respectively. 


$$
\begin{aligned}
& \varepsilon_{/ /}=\left(1-\phi_{M}\right)+\varepsilon \phi_{M}\{1+<N>(\varepsilon-1)\}^{-1}, \\
& \varepsilon_{\perp}=\left(1-\phi_{M}\right)+2 \varepsilon \phi_{M}\{(\varepsilon+1)-<N>(\varepsilon-1)\}^{-1},
\end{aligned}
$$

where $\varepsilon$ is the relative dielectric constant of the colloidal particle with respect to the solvent. Here the second and higher order terms with respect to $\langle N\rangle(\varepsilon-1)$ are neglected. Consequently, the phase difference $\theta$ is approximated as

$$
\theta=C(1 / 3-<N>),
$$

where $\mathrm{C}$ is a constant and the approximation

$$
\sqrt{\varepsilon_{\| /}}-\sqrt{\varepsilon_{\perp}}=\left(\varepsilon_{/ /}-\varepsilon_{\perp}\right) / 2
$$

is used.

In reference [13], the phenomenological interpretation of the generalized Curie-Weiss law was proposed using the Landau's phase transition theorem of the second order[15]. Here we discuss this law from a mathematical view point. Connecting eqs.(6) and (12), and using eqs. (8) and (9), the equation

$$
1 /\left\{\mathrm{T}-\mathrm{T}_{\mathrm{O}}(\mathrm{H})\right\}=g(\mathrm{H}) \sum_{\mathrm{k}=2}^{\infty} \mathrm{kv} \mathrm{v}_{\mathrm{O}} \nu_{\mathrm{k}}\left(1 / 3-\mathrm{N}_{\mathrm{k}}\right) \text {, }
$$

is obtained, where $\mathrm{g}(\mathrm{H})$ is the function of $\mathrm{H}$ but independent of $\mathrm{T}$. Here $\mathrm{k}$ starts from 2 because $\mathrm{N}_{1}=1 / 3$. In the right hand side of eq. (14), only the factors $L(k=1,2, \ldots)$ are the functions of $T$. In addition, they are smooth decreasing functions of $T$. On the other hand the left hand side of eq. (14) has a singular point at the finite temperature $\mathrm{T}=\mathrm{T}(\mathrm{H})$. The origin of the appearance of the singular point at the finite temperature is the summation of the infinite number of the terms of the series of the smooth functions with respect to $T$. The left hand side of eq.(14) is also expanded in the infinite series and the eq.(14) is rewritten as

$$
\left(1 / T_{0}\right) \sum_{k=2}^{\infty}\left(T_{0} / T\right)^{k-1}=g(H) \sum_{k=2}^{\infty} k v_{0} \nu_{k}\left(1 / 3-N_{k}\right) \text {. }
$$

Some physical interpretations are possible with regard to the eq.(15). The definite physical model, however, is not obtained in the present state of knowledge.

\section{References}

1. R. E. Rosensweig: Encyclopaedic dictionary of physics Suppliment Vol.4 p.1.

2. R. V. Mehta: J. Colloid Interface Sci. 42 (1973) 165.

3. G. Goldberg, J. Hansford and P. J. van Heerden: J. Appl. Phys. 42 (1971) 3874.

4. P. A. Martinet: Rheol. Acta 13 (1974) 260.

5. W. E. I. Haas and J. E. Adams: Appl. Phys. Lett. 27 (1975) 57.

6. P. C. Scholten: IEEE Trans. Mag. MAG-11 (1975) 1400.

7. C. F. Hayes, S. R. Hwang: J. Colloid Interface Sci. 60 (1977) 443.

8. Yu. N. Skibin, V. V. Chekanov and Yu. L. Raikher: Zh. Eksp. Teor. Fiz. 72 (1977) 949.

9. U. Neitzel and K. Baerner: Phys. Lett. 63A (1977) 327.

10. H. W. Davies and J. P. Llewellyn: J. Phys. D 12 (1979) 311.

11. S. Taketomi: Jpn. J. Appl. Phys. 22 (1983) 1137.

12. S. Chikazumi, S. Taketomi, M. Ukita, M. Mizukami, H. Miyajima, M. Setogawa and Y. Kurihara: J. Mag. Mag. Mat. 65 (1987) 245.

13. S. Taketomi, M. Ukita, M. Mizukami, H. Miyajima and S. Chikazumi: submitted to J. Phys. Soc. Jpn.

14. R. Gans: Ann. Physik 37 (1912) 881, ibid 47 (1915) 270.

15. I. D. Landau and E. M. Lifschitz: Statistical Physics (Pergamon Press Oxford ) (1958). 